\title{
A living eukaryotic auto-cementation kit from surface display of silica binding peptides on Yarrowia lipolytica
}

\author{
Supplementary data
}

\section{Promoters and ORF constructed in this project}

\section{LIP2prepro signal peptide}

ATGAAGCTTTCCACCATCCTTTTCACAGCCTGCGCTACCCTGGCTGCCGCCCTCCCTTCCCCCATCACTCCTTCTGAGGCC GCAGTTCTCCAGAAGCGA

\section{Ylcwp3 anchor peptide}

CTCGGCTTTGCCGCTCGAGCTGTCTTCGAAGGTGGCTCTTCTTCCGCCGCTGCTCCCACCTCTTCCTCCGCTGCTTCCCAT GCCGCCTCTTCCGCTGCCGCCTCTGCTTCTCACGCTGCTTCTTCTGCTGCTGCTTCCAAGGCTTCTTCTGCTGTTGCCGCC CCCAAGTCCGAGGCCGCTGCTGGTGCTCACGCCACTGCCGGTGCCATTGTCTCCCAGATCAATGATGGCCAGATCCAGGC TCCCCACTCCACCGGCCCTGCCCAGGCCCCCAAGGCCTCTGCTCCTCCCGCCCAGGCCAACGGCGCTGCCACCCTTGGT GTCTCTGCTGTTGCCGGTGCTGTTGCCGTCGCCATGCTTTTCTAA

\section{Si-tag $1+2+3$}

ATGGCAGTTGTTAAATGTAAACCGACATCTCCGGGTCGTCGCCACGTAGTTAAAGTGGTTAACCCTGAGCTGCACAAGGG CAAACCTTTTGCTCCGTTGCTGGAAAAAAACAGCAAATCCGGTGGTCGTAACAACAATGGCCGTATCACCACTCGTCATA TCGGTGGTGGCCACAAGCAGGCTTACCGTATTGTTGACTTCAAACGCAACAAAGACGGTATCCCGGCAGTTGTTGAACG TCTTGAGTACGATCCGAACCGTTCCGCGAACATCGCGCTGGTTCTGTACAAAGACGGTGAACGCCGTTACATCCTGGCCC CTAAAGGCCTGAAAGCTGGCGACCAGATTCAGTCTGGCGTTGATGCTGCAATCAAACCAGGTAACACCCTGCCGATGCG CAACATCCCGGTTGGTTCTACTGTTCATAACGTAGAAATGAAACCAGGTAAAGGCGGTCAGCTGGCACGTTCCGCTGGTA CTTACGTTCAGATCGTTGCTCGTGATGGTGCTTATGTCACCCTGCGTCTGCGTTCTGGTGAAATGCGTAAAGTAGAAGCAG ACTGCCGTGCAACTCTGGGCGAAGTTGGCAATGCTGAGCATATGCTGCGCGTTCTGGGTAAAGCAGGTGCTGCACGCTG GCGTGGTGTTCGTCCGACCGTTCGCGGTACCGCGATGAACCCGGTAGACCACCCACATGGTGGTGGTGAAGGTCGTAAC TTTGGTAAGCACCCGGTAACTCCGTGGGGCGTTCAGACCAAAGGTAAGAAGACCCGCAGCAACAAGCGTACTGATAAAT TCATCGTACGTCGCCGTAGCAAATAA

\section{Mcfp-3 flocculating protein}

ATGAATAAATTCAGTGTCACAGTTTTGCTGGCTTTAGTCCTTATTGGATTTTTTGCCGTTCAGAGTGACGCAGGTTATGGTT 
ATGATCTAGGATATAATGCACCATGGCCATACAACAATGGTTACTATGGCTATAATGGATACAATGGATATCACGGACGTTAT GGCTGGAATAAAGGCTGGAATAACGGTCCATGGGGAGGATCATATTATGGAAACAAAGGCTATTTGTATTAG

\title{
Recombinant sequence:
}

\section{LIP2prepro-6xHis-Mcfp-3}

\begin{abstract}
ATGAAGCTTTCCACCATCCTTTTCACAGCCTGCGCTACCCTGGCTGCCGCCCTCCCTTCCCCCATCACTCCTTCTGAGGC CGCAGTTCTCCAGAAGCGAGGATCCCACCACCACCACCACCACATGAATAAATTCAGTGTCACAGTTTTGCTGGCTTTA GTCCTTATTGGATTTTTTGCCGTTCAGAGTGACGCAGGTTATGGTTATGATCTAGGATATAATGCACCATGGCCATACAA CAATGGTTACTATGGCTATAATGGATACAATGGATATCACGGACGTTATGGCTGGAATAAAGGCTGGAATAACGGTCC ATGGGGAGGATCATATTATGGAAACAAAGGCTATTTGTATGTCGACGGCGGCGGCGGCTCTGGCGGCGGCGGCTCTGG CGGCGGCGGCTCTCATATGCTCGGCTTTGCCGCTCGAGCTGTCTTCAAGGTGGCTCTTCTTCCGCCGCTGCTCCCACCTC TTCCTCCGCTGCTTCCCATGCCGCCTCTTCCGCTGCCGCCTCTGCTTCTCACGCTGCTTCTTCTGCTGCTGCTTCCAAGGC TTCTTCTGCTGTTGCCGCCCCCAAGTCCGAGGCCGCTGCTGGTGCTCACGCCACTGCCGGTGCCATTGTCTCCCAGATCA ATGATGGCCAGATCCAGGCTCCCCACTCCACCGGCCCTGCCCAGGCCCCCAAGGCCTCTGCTCCTCCCGCCCAGGCCAA CGGCGCTGCCACCCTTGGTGTCTCTGCTGTTGCCGGTGCTGTTGCCGTCGCCATGCTTTTCTAA
\end{abstract}

\section{LIP2prepro-6xHis-Si-tag1-Ylcwp3}

ATGAAGCTTTCCACCATCCTTTTCACAGCCTGCGCTACCCTGGCTGCCGCCCTCCCTTCCCCCATCACTCCTTCTGAGGC CGCAGTTCTCCAGAAGCGAGGATCCCACCACCACCACCACCACATGGCAGTTGTTAAATGTAAACCGACATCTCCGGGT CGTCGCCACGTAGTTAAAGTGGTTAACCCTGAGCTGCACAAGGGCAAACCTTTTGCTCCGTTGCTGGAAAAAAACAGCA AATCCGGTGGTCGTAACAACAATGGCCGTATCACCACTCGTCATATCGGTGGTGGCCACAAGCAGGTCGACGGCGGCG GCGGCTCTGGCGGCGGCGGCTCTGGCGGCGGCGGCTCTCATATGCTCGGCTTTGCCGCTCGAGCTGTCTTCAAGGTGGC TCTTCTTCCGCCGCTGCTCCCACCTCTTCCTCCGCTGCTTCCCATGCCGCCTCTTCCGCTGCCGCCTCTGCTTCTCACGCT GCTTCTTCTGCTGCTGCTTCCAAGGCTTCTTCTGCTGTTGCCGCCCCCAAGTCCGAGGCCGCTGCTGGTGCTCACGCCAC TGCCGGTGCCATTGTCTCCCAGATCAATGATGGCCAGATCCAGGCTCCCCACTCCACCGGCCCTGCCCAGGCCCCCAAG GCCTCTGCTCCTCCCGCCCAGGCCAACGGCGCTGCCACCCTTGGTGTCTCTGCTGTTGCCGGTGCTGTTGCCGTCGCCAT GCTTTTCTAA

\section{LIP2prepro-6xHis-Si-tag2-Ylcwp3}

ATGAAGCTTTCCACCATCCTTTTCACA GCCTGCGCTACCCTGGCTGCCGCCCTCCCTTCCCCCATCACTCCTTCTGAGGC
CGCAGTTCTCCAGAAGCGAGGATCCAACCACCACCACCACCACAGCTTACCGTATTGTTGACTTCAAACGCAACAAAGA
CGGTATCCCGGCAGTTGTTGAACGTCTTGAGTACGATCCGAACCGTTCCGCGAACATCGCGCTGGTTCTGTACAAAGAC
GGTGAACGCCGTTACATCCTGGCCCCTAAAGGCCTGAAAGCTGGCGACCAGATTCAGTCTGGCGTTGATGCTGCAATCA
AACCAGGTAACACCCTGCCGATGCGCAACATCCCGGTTGGTTCTACTGTTCATAACGTAGAAATGAAACCAGGTAAAG
GCGGTCAGCTGGCACGTTCCGCTGGTACTTACGTTCAGATCGTTGCTCGTGATGGTGCTTATGTCACCCTGCGTCTGCGT
TCTGGTGAAATGCGTAAAGTAGAAGCAGACTGCCGTGCAACTCTGGGCGAAGTTGGCAATGCTGAGCATATGCTGGTC
GACGGCGGCGGCGGCTCTGGCGGCGGCGGCTCTGGCGGCGGCGGCTCTCATATGCTCGGCTTTGCCGCTCGAGCTGTCT 
TCAAGGTGGCTCTTCTTCCGCCGCTGCTCCCACCTCTTCCTCCGCTGCTTCCCATGCCGCCTCTTCCGCTGCCGCCTCTGC TTCTCACGCTGCTTCTTCTGCTGCTGCTTCCAAGGCTTCTTCTGCTGTTGCCGCCCCCAAGTCCGAGGCCGCTGCTGGTGC TCACGCCACTGCCGGTGCCATTGTCTCCCAGATCAATGATGGCCAGATCCAGGCTCCCCACTCCACCGGCCCTGCCCAG GCCCCCAAGGCCTCTGCTCCTCCCGCCCAGGCCAACGGCGCTGCCACCCTTGGTGTCTCTGCTGTTGCCGGTGCTGTTGC CGTCGCCATGCTTTTCTAA

\title{
LIP2prepro-6xHis-Si-tag3-Ylcwp3
}

\begin{abstract}
ATGAAGCTTTCCACCATCCTTTTCACAGCCTGCGCTACCCTGGCTGCCGCCCTCCCTTCCCCCATCACTCCTTCTGAGGC CGCAGTTCTCCAGAAGCGAGGATCCCACCACCACCACCACCACCGCGTTCTGGGTAAAGCAGGTGCTGCACGCTGGCG TGGTGTTCGTCCGACCGTTCGCGGTACCGCGATGAACCCGGTAGACCACCCACATGGTGGTGGTGAAGGTCGTAACTTT GGTAAGCACCCGGTAACTCCGTGGGGCGTTCAGACCAAAGGTAAGAAGACCCGCAGCAACAAGCGTACTGATAAATTC ATCGTACGTCGCCGTAGCAAATAAGTCGACGGCGGCGGCGGCTCTGGCGGCGGCGGCTCTGGCGGCGGCGGCTCTCAT ATGCTCGGCTTTGCCGCTCGAGCTGTCTTCAAGGTGGCTCTTCTTCCGCCGCTGCTCCCACCTCTTCCTCCGCTGCTTCCC ATGCCGCCTCTTCCGCTGCCGCCTCTGCTTCTCACGCTGCTTCTTCTGCTGCTGCTTCCAAGGCTTCTTCTGCTGTTGCCG CCCCCAAGTCCGAGGCCGCTGCTGGTGCTCACGCCACTGCCGGTGCCATTGTCTCCCAGATCAATGATGGCCAGATCCA GGCTCCCCACTCCACCGGCCCTGCCCAGGCCCCCAAGGCCTCTGCTCCTCCCGCCCAGGCCAACGGCGCTGCCACCCTT GGTGTCTCTGCTGTTGCCGGTGCTGTTGCCGTCGCCATGCTTTTCTAA
\end{abstract}

\section{LIP2prepro-6xHis-Si-tag1+2-Ylcwp3}

ATGAAGCTTTCCACCATCCTTTTCACAGCCTGCGCTACCCTGGCTGCCGCCCTCCCTTCCCCCATCACTCCTTCTGAGGC CGCAGTTCTCCAGAAGCGAGGATCCCACCACCACCACCACCACATGGCAGTTGTTAAATGTAAACCGACATCTCCGGGT CGTCGCCACGTAGTTAAAGTGGTTAACCCTGAGCTGCACAAGGGCAAACCTTTTGCTCCGTTGCTGGAAAAAAACAGCA AATCCGGTGGTCGTAACAACAATGGCCGTATCACCACTCGTCATATCGGTGGTGGCCACAAGCAGGCTTACCGTATTGTTG ACTTCAAACGCAACAAAGACGGTATCCCGGCAGTTGTTGAACGTCTTGAGTACGATCCGAACCGTTCCGCGAACATCGC GCTGGTTCTGTACAAAGACGGTGAACGCCGTTACATCCTGGCCCCTAAAGGCCTGAAAGCTGGCGACCAGATTCAGTCT GGCGTTGATGCTGCAATCAAACCAGGTAACACCCTGCCGATGCGCAACATCCCGGTTGGTTCTACTGTTCATAACGTAGA AATGAAACCAGGTAAAGGCGGTCAGCTGGCACGTTCCGCTGGTACTTACGTTCAGATCGTTGCTCGTGATGGTGCTTATG TCACCCTGCGTCTGCGTTCTGGTGAAATGCGTAAAGTAGAAGCAGACTGCCGTGCAACTCTGGGCGAAGTTGGCAATGC TGAGCATATGCTGGTCGACGGCGGCGGCGGCTCTGGCGGCGGCGGCTCTGGCGGCGGCGGCTCTCATATGCTCGGCTTT GCCGCTCGAGCTGTCTTCAAGGTGGCTCTTCTTCCGCCGCTGCTCCCACCTCTTCCTCCGCTGCTTCCCATGCCGCCTCTT CCGCTGCCGCCTCTGCTTCTCACGCTGCTTCTTCTGCTGCTGCTTCCAAGGCTTCTTCTGCTGTTGCCGCCCCCAAGTCCG AGGCCGCTGCTGGTGCTCACGCCACTGCCGGTGCCATTGTCTCCCAGATCAATGATGGCCAGATCCAGGCTCCCCACTC CACCGGCCCTGCCCAGGCCCCCAAGGCCTCTGCTCCTCCCGCCCAGGCCAACGGCGCTGCCACCCTTGGTGTCTCTGCT GTTGCCGGTGCTGTTGCCGTCGCCATGCTTTTCTAA

\section{LIP2prepro-6xHis-Si-tag2+3-Ylcwp3}

ATGAAGCTTTCCACCATCCTTTTCACAGCCTGCGCTACCCTGGCTGCCGCCCTCCCTTCCCCCATCACTCCTTCTGAGGC 
CGCAGTTCTCCAGAAGCGAGGATCCCACCACCACCACCACCACGCTTACCGTATTGTTGACTTCAAACGCAACAAAGAC GGTATCCCGGCAGTTGTTGAACGTCTTGAGTACGATCCGAACCGTTCCGCGAACATCGCGCTGGTTCTGTACAAAGACGG TGAACGCCGTTACATCCTGGCCCCTAAAGGCCTGAAAGCTGGCGACCAGATTCAGTCTGGCGTTGATGCTGCAATCAAAC CAGGTAACACCCTGCCGATGCGCAACATCCCGGTTGGTTCTACTGTTCATAACGTAGAAATGAAACCAGGTAAAGGCGGT CAGCTGGCACGTTCCGCTGGTACTTACGTTCAGATCGTTGCTCGTGATGGTGCTTATGTCACCCTGCGTCTGCGTTCTGGT GAAATGCGTAAAGTAGAAGCAGACTGCCGTGCAACTCTGGGCGAAGTTGGCAATGCTGAGCATATGCTGCGCGTTCTGG GTAAAGCAGGTGCTGCACGCTGGCGTGGTGTTCGTCCGACCGTTCGCGGTACCGCGATGAACCCGGTAGACCACCCACA TGGTGGTGGTGAAGGTCGTAACTTTGGTAAGCACCCGGTAACTCCGTGGGGCGTTCAGACCAAAGGTAAGAAGACCCGC AGCAACAAGCGTACTGATAAATTCATCGTACGTCGCCGTAGCAAATAAGTCGACGGCGGCGGCGGCTCTGGCGGCGGCG GCTCTGGCGGCGGCGGCTCTCATATGCTCGGCTTTGCCGCTCGAGCTGTCTTCAAGGTGGCTCTTCTTCCGCCGCTGCTC CCACCTCTTCCTCCGCTGCTTCCCATGCCGCCTCTTCCGCTGCCGCCTCTGCTTCTCACGCTGCTTCTTCTGCTGCTGCTTC CAAGGCTTCTTCTGCTGTTGCCGCCCCCAAGTCCGAGGCCGCTGCTGGTGCTCACGCCACTGCCGGTGCCATTGTCTCCC AGATCAATGATGGCCAGATCCAGGCTCCCCACTCCACCGGCCCTGCCCAGGCCCCCAAGGCCTCTGCTCCTCCCGCCCA GGCCAACGGCGCTGCCACCCTTGGTGTCTCTGCTGTTGCCGGTGCTGTTGCCGTCGCCATGCTTTTCTAA

\section{LIP2prepro-6xHis-Si-tag1+3-Ylcwp3}

ATGAAGCTTTCCACCATCCTTTTCACAGCCTGCGCTACCCTGGCTGCCGCCCTCCCTTCCCCCATCACTCCTTCTGAGGC CGCAGTTCTCCAGAAGCGAGGATCCCACCACCACCACCACCACATGGCAGTTGTTAAATGTAAACCGACATCTCCGGGT CGTCGCCACGTAGTTAAAGTGGTTAACCCTGAGCTGCACAAGGGCAAACCTTTTGCTCCGTTGCTGGAAAAAAACAGCA AATCCGGTGGTCGTAACAACAATGGCCGTATCACCACTCGTCATATCGGTGGTGGCCACAAGCAGCGCGTTCTGGGTAAA GCAGGTGCTGCACGCTGGCGTGGTGTTCGTCCGACCGTTCGCGGTACCGCGATGAACCCGGTAGACCACCCACATGGTG GTGGTGAAGGTCGTAACTTTGGTAAGCACCCGGTAACTCCGTGGGGCGTTCAGACCAAAGGTAAGAAGACCCGCAGCA ACAAGCGTACTGATAAATTCATCGTACGTCGCCGTAGCAAATAAGTCGACGGCGGCGGCGGCTCTGGCGGCGGCGGCTCT GGCGGCGGCGGCTCTCATATGCTCGGCTTTGCCGCTCGAGCTGTCTTCAAGGTGGCTCTTCTTCCGCCGCTGCTCCCACC TCTTCCTCCGCTGCTTCCCATGCCGCCTCTTCCGCTGCCGCCTCTGCTTCTCACGCTGCTTCTTCTGCTGCTGCTTCCAAG GCTTCTTCTGCTGTTGCCGCCCCCAAGTCCGAGGCCGCTGCTGGTGCTCACGCCACTGCCGGTGCCATTGTCTCCCAGAT CAATGATGGCCAGATCCAGGCTCCCCACTCCACCGGCCCTGCCCAGGCCCCCAAGGCCTCTGCTCCTCCCGCCCAGGCC AACGGCGCTGCCACCCTTGGTGTCTCTGCTGTTGCCGGTGCTGTTGCCGTCGCCATGCTTTTCTAA

\section{LIP2prepro-6xHis-Si-tag1+GSlinker+3-Ylcwp3}

ATGAAGCTTTCCACCATCCTTTTCACAGCCTGCGCTACCCTGGCTGCCGCCCTCCCTTCCCCCATCACTCCTTCTGAGGC CGCAGTTCTCCAGAAGCGAGGATCCCACCACCACCACCACCACATGGCAGTTGTTAAATGTAAACCGACATCTCCGGGT CGTCGCCACGTAGTTAAAGTGGTTAACCCTGAGCTGCACAAGGGCAAACCTTTTGCTCCGTTGCTGGAAAAAAACAGCA AATCCGGTGGTCGTAACAACAATGGCCGTATCACCACTCGTCATATCGGTGGTGGCCACAAGCAGGGTGGCGGAGGTTCA CGCGTTCTGGGTAAAGCAGGTGCTGCACGCTGGCGTGGTGTTCGTCCGACCGTTCGCGGTACCGCGATGAACCCGGTAG ACCACCCACATGGTGGTGGTGAAGGTCGTAACTTTGGTAAGCACCCGGTAACTCCGTGGGGCGTTCAGACCAAAGGTAA GAAGACCCGCAGCAACAAGCGTACTGATAAATTCATCGTACGTCGCCGTAGCAAATAAGTCGACGGCGGCGGCGGCTCT GGCGGCGGCGGCTCTGGCGGCGGCGGCTCTCATATGCTCGGCTTTGCCGCTCGAGCTGTCTTCAAGGTGGCTCTTCTTCC GCCGCTGCTCCCACCTCTTCCTCCGCTGCTTCCCATGCCGCCTCTTCCGCTGCCGCCTCTGCTTCTCACGCTGCTTCTTCT 
GCTGCTGCTTCCAAGGCTTCTTCTGCTGTTGCCGCCCCCAAGTCCGAGGCCGCTGCTGGTGCTCACGCCACTGCCGGTGC CATTGTCTCCCAGATCAATGATGGCCAGATCCAGGCTCCCCACTCCACCGGCCCTGCCCAGGCCCCCAAGGCCTCTGCT CCTCCCGCCCAGGCCAACGGCGCTGCCACCCTTGGTGTCTCTGCTGTTGCCGGTGCTGTTGCCGTCGCCATGCTTTTCTA A

\section{LIP2prepro-6xHis-Si-tag1+2+3-Ylcwp3}

ATGAAGCTTTCCACCATCCTTTTCACAGCCTGCGCTACCCTGGCTGCCGCCCTCCCTTCCCCCATCACTCCTTCTGAGGC CGCAGTTCTCCAGAAGCGAGGATCCCACCACCACCACCACCACATGGCAGTTGTTAAATGTAAACCGACATCTCCGGGT CGTCGCCACGTAGTTAAAGTGGTTAACCCTGAGCTGCACAAGGGCAAACCTTTTGCTCCGTTGCTGGAAAAAAACAGCA AATCCGGTGGTCGTAACAACAATGGCCGTATCACCACTCGTCATATCGGTGGTGGCCACAAGCAGGCTTACCGTATTGTTG ACTTCAAACGCAACAAAGACGGTATCCCGGCAGTTGTTGAACGTCTTGAGTACGATCCGAACCGTTCCGCGAACATCGC GCTGGTTCTGTACAAAGACGGTGAACGCCGTTACATCCTGGCCCCTAAAGGCCTGAAAGCTGGCGACCAGATTCAGTCT GGCGTTGATGCTGCAATCAAACCAGGTAACACCCTGCCGATGCGCAACATCCCGGTTGGTTCTACTGTTCATAACGTAGA AATGAAACCAGGTAAAGGCGGTCAGCTGGCACGTTCCGCTGGTACTTACGTTCAGATCGTTGCTCGTGATGGTGCTTATG TCACCCTGCGTCTGCGTTCTGGTGAAATGCGTAAAGTAGAAGCAGACTGCCGTGCAACTCTGGGCGAAGTTGGCAATGC TGAGCATATGCTGCGCGTTCTGGGTAAAGCAGGTGCTGCACGCTGGCGTGGTGTTCGTCCGACCGTTCGCGGTACCGCGA TGAACCCGGTAGACCACCCACATGGTGGTGGTGAAGGTCGTAACTTTGGTAAGCACCCGGTAACTCCGTGGGGCGTTCA GACCAAAGGTAAGAAGACCCGCAGCAACAAGCGTACTGATAAATTCATCGTACGTCGCCGTAGCAAATAAGTCGACGGC GGCGGCGGCTCTGGCGGCGGCGGCTCTGGCGGCGGCGGCTCTCATATGCTCGGCTTTGCCGCTCGAGCTGTCTTCAAGG TGGCTCTTCTTCCGCCGCTGCTCCCACCTCTTCCTCCGCTGCTTCCCATGCCGCCTCTTCCGCTGCCGCCTCTGCTTCTCA CGCTGCTTCTTCTGCTGCTGCTTCCAAGGCTTCTTCTGCTGTTGCCGCCCCCAAGTCCGAGGCCGCTGCTGGTGCTCACG CCACTGCCGGTGCCATTGTCTCCCAGATCAATGATGGCCAGATCCAGGCTCCCCACTCCACCGGCCCTGCCCAGGCCCC CAAGGCCTCTGCTCCTCCCGCCCAGGCCAACGGCGCTGCCACCCTTGGTGTCTCTGCTGTTGCCGGTGCTGTTGCCGTCG CCATGCTTTTCTAA 


\section{Modeling Simulation}

With the benefit of synthetic biology, we built up our kit, Euk.cement, with some characterized parts to carry out the mission of sand solidification. However, in order to achieve these goals, we have to determine some factors. First of all, we have to make sure whether the light induction system could be able to switch efficiently to control the expression of target proteins. Secondly, we need to know what strategy we should take in practice to make full use of our product. Moreover, the amount of Si-tag and Mcfp-3 produced by our strains in the end must be considered.

To ensure that Euk.cement is capable, we built a Delay Differential Equations (DDE) model based on Michaelis-Menten equation and Chemical reaction rate equation, which includes three parts, to get the insight of how each part works cooperatively. The Matlab code of the DDEs model is listed as below:

-light_induction_system

function light_induction_system()

$\%$ this program is used to simulate the biological process of Light Induction System

history=zeros $(12,1)$;

tau=0.1;

$\%$ initiate the values of every subjects and tau

dark_1=dde23(@exposed_to_darkness,tau,history,[-2000,0]);

light_1=dde23(@exposed_to_light,tau,dark_1.y(:,end),[0,2000]);

dark_2=dde23(@exposed_to_darkness,tau,light_1.y(:,end),[2000,4000]);

$\%$ calculate the values of every subjects in the DDE model

plot(dark_1.x,dark_1.y(8,:),'r',dark_1.x,dark_1.y(11,:),'b','LineWidth',2.5);

hold on

stem(0,5,'k','Marker','none','LineWidth',2.5);

hold on

stem(2000,5,'m','Marker','none','LineWidth',2.5);

hold on

legend('Si-Tag','Mcfp-3','Light ON','Light OFF');

plot(light_1.x,light_1.y(8,:),'r',light_1.x,light_1.y(11,:),'b','LineWidth',2.5);

hold on

plot(dark_2.x,dark_2.y(8,:),'r',dark_2.x,dark_2.y(11,:),'b','LineWidth',2.5);

hold on

$x \lim ([-20004000])$;

hold on

ylim([0 5]);

title('Simulation of Light Induction System','fontsize',16,'fontweight','b'); 
xlabel('time /min','fontsize',16,'fontweight','b');

ylabel('expression level of Si-tag \& Mcfp-3','fontsize',16,'fontweight','b');

set(gca,'xtick',[-2000 -1000 0100020003000 4000]);

set(gca,'FontName','Times New Roman','FontSize',12);

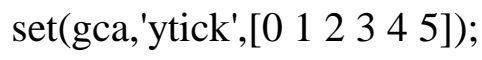

set(gcf,'Position',[50 351300 800]);

hold off

$\%$ plot the figure

exposed_to_darkness

function dy=exposed_to_darkness $(\mathrm{t}, \mathrm{y}, \mathrm{Z})$

$\%$ the DDE model in the dark condition

$\% \mathrm{y}(1)$ as mCRY2_BD

$\% \mathrm{y}(2)$ as CRY2_BD

$\% \mathrm{y}(3)$ as (active)CRY2_BD

$\% \mathrm{y}(4)$ as mCIB1_AD

$\% \mathrm{y}(5)$ as CIB1_AD

$\% \mathrm{y}(6)$ as (active)CIB1_AD

$\% \mathrm{y}(7)$ as mSi_Tag

$\%$ y(8) as Si_Tag

$\% \mathrm{y}(9)$ as outerSi_Tag

$\% \mathrm{y}(10)$ as mMcfp-3

$\% \mathrm{y}(11)$ as Mcfp-3

$\% \mathrm{y}(12)$ as outerMcfp-3

$\mathrm{dy}=\mathrm{zeros}(12,1)$;

$\mathrm{dy}(1)=4.25 * 16-(180 / 12) * \mathrm{y}(1)$;

$\operatorname{dy}(2)=8.31 * y(1)-y(2)$;

$\operatorname{dy}(3)=0-0.0000355 * y(3)$;

$\mathrm{dy}(4)=7.82 * 16-(180 / 13) * \mathrm{y}(4)$;

$\mathrm{dy}(5)=6.65 * \mathrm{y}(4)-\mathrm{y}(5)$;

$\mathrm{dy}(6)=0-0.0017 * \mathrm{y}(6)$;

$\operatorname{dy}(7)=2.02 * 16 *(\mathrm{y}(3) /(\mathrm{y}(3)+4.93)) *(\mathrm{Z}(6,1) /(\mathrm{Z}(6,1)+1.04))+0.2 *(16-(\mathrm{y}(3) /(\mathrm{y}(3)+4.93)) *(\mathrm{Z}(6,1) /(\mathrm{Z}(6$,

$1)+1.04)))-(240 / 13) * \mathrm{y}(7)$;

$\operatorname{dy}(8)=25.7 * y(7)-(18 / 48) * y(8)-13.7 * y(8)$;

$\operatorname{dy}(9)=13.7 * y(8)$

$\mathrm{dy}(10)=0.581 * 16 *(\mathrm{y}(3) /(\mathrm{y}(3)+4.93)) *(\mathrm{Z}(6,1) /(\mathrm{Z}(6,1)+1.04))+0.056 *(16-(\mathrm{y}(3) /(\mathrm{y}(3)+4.93)) *(\mathrm{Z}(6,1) /($ $\mathrm{Z}(6,1)+1.04)))-(240 / 13) * \mathrm{y}(10)$;

$\operatorname{dy}(11)=31.2 * y(10)-0.25 * \mathrm{y}(11)-15.2 * \mathrm{y}(11)$;

$\operatorname{dy}(12)=15.2 * y(11)$; 


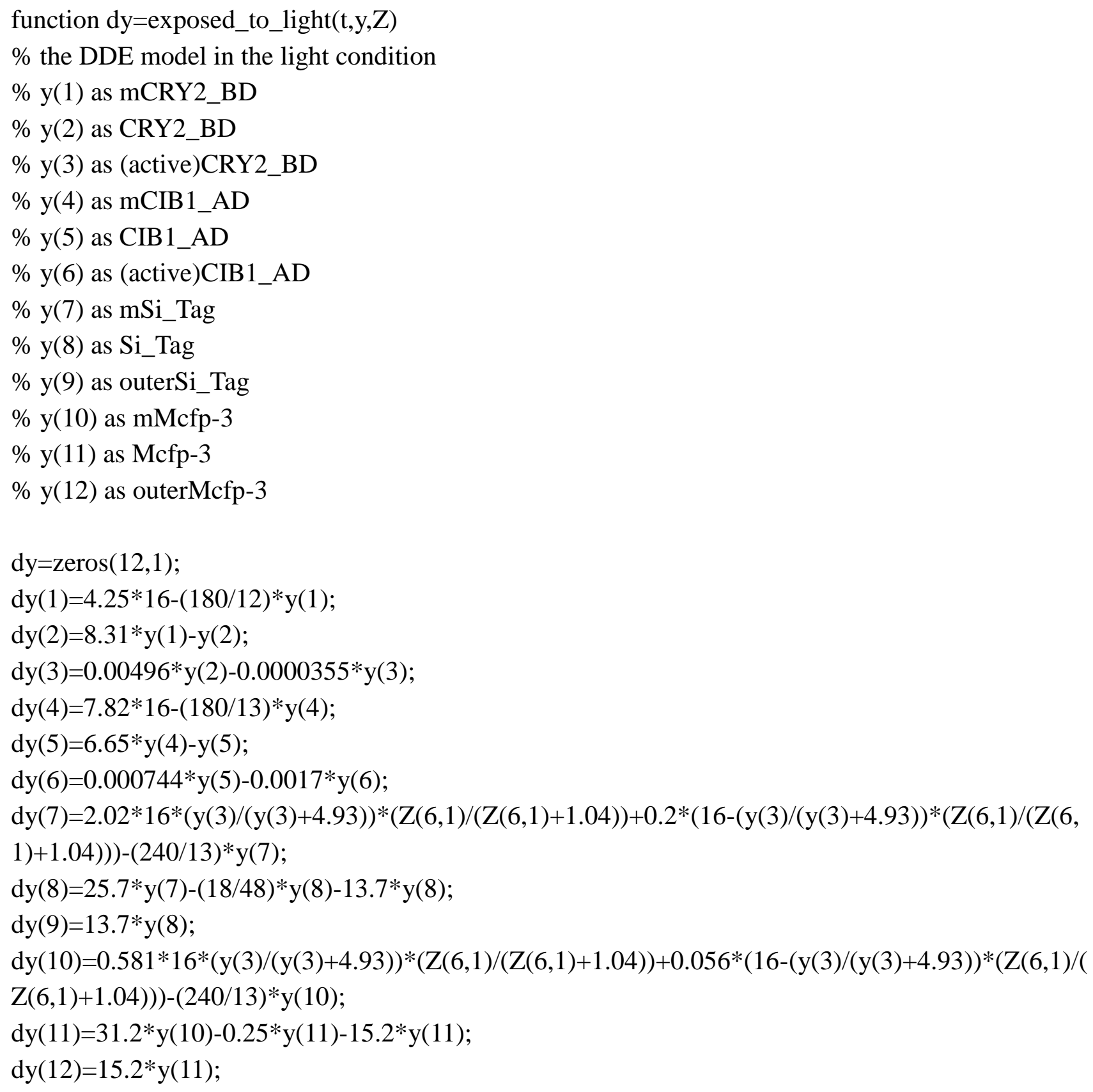

Note:

(1) 'light_induction_system'is the main program which plots the figure of the whole process of the biological system.

(2) 'exposed_to_light' is a set of delay differential equations which describes the biological system exposed to light.

(3) 'exposed_to_darkness' is a set of delay differential equations which describes the biological system exposed to darkness.

\section{Parameters}

The description of parameters, their values and the references involved in this DDE model are listed 
in a table:

\begin{tabular}{|c|c|c|c|c|}
\hline parameter & Description & Value & dimensions & references \\
\hline copynum $_{\mathrm{pRNH} 120}$ & copy number of plasmid pRMH120 & $14^{\sim} 19$ & \multirow{2}{*}{ number } & \multirow{2}{*}{$\begin{array}{l}\text { estimated } \\
\text { according } \\
\text { to Addgene }\end{array}$} \\
\hline copynum $_{\mathrm{pZs} 3}$ & copy number of plasmid pZS3 & $12^{\sim} 17$ & & \\
\hline $\operatorname{trc}_{1}$ & rate of mCRY2_BD transcription & $7.82 \mathrm{E}+00$ & $\mathrm{nM} / \mathrm{h}$ & \multirow[t]{2}{*}[1]{$[3]$} \\
\hline $\operatorname{trc}_{2}$ & rate of mCIB1_AD transcription & 4. $25 \mathrm{E}+00$ & $\mathrm{nM} / \mathrm{h}$ & \\
\hline $\operatorname{trc}_{3}$ & rate of $\mathrm{mSi}$ Tag transcription & $2.02 \mathrm{E}+00$ & $\mathrm{nM} / \mathrm{h}$ & \multirow[t]{4}{*}{ estimated } \\
\hline $\operatorname{trc}_{4}$ & $\begin{array}{l}\text { basic rate of } \mathrm{mSi}_{-} \text {Tag } \\
\text { transcription }\end{array}$ & $\sim 2.00 \mathrm{E}-01$ & $\mathrm{nM} / \mathrm{h}$ & \\
\hline $\operatorname{trc}_{5}$ & rate of mMcfp-3 transcription & $5.81 \mathrm{E}-01$ & $\mathrm{nM} / \mathrm{h}$ & \\
\hline $\operatorname{trc}_{6}$ & $\begin{array}{l}\text { basic rate of mMcfp-3 } \\
\text { transcription }\end{array}$ & $5.56 \mathrm{E}-02$ & $\mathrm{nM} / \mathrm{h}$ & \\
\hline $\operatorname{tr} l_{1}$ & rate of CRY2_BD translation & $8.31 \mathrm{E}+00$ & $1 / \mathrm{nM} * \mathrm{~h}$ & \multirow[t]{2}{*}[1]{$[3]$} \\
\hline $\operatorname{trl} l_{2}$ & rate of CIB1_AD translation & $6.65 \mathrm{E}+00$ & $1 / \mathrm{nM} * \mathrm{~h}$ & \\
\hline $\operatorname{trl} l_{4}$ & rate of $\mathrm{Si}$ Tag translation & $2.57 \mathrm{E}+01$ & $1 / \mathrm{nM} * \mathrm{~h}$ & [4] \\
\hline $\operatorname{tr} 1_{5}$ & rate of Mcfp-3 translation & 3. $12 \mathrm{E}+01$ & $1 / \mathrm{nM} * \mathrm{~h}$ & {$[5]$} \\
\hline $\operatorname{trf} f_{1}$ & $\begin{array}{c}\text { rate of photoactivation of } \\
\text { CRY2_BD }\end{array}$ & 4. $96 \mathrm{E}-03$ & $\mathrm{~m} 2 / \mathrm{um}$ & \multirow[t]{4}{*}[1]{$[3]$} \\
\hline $\operatorname{trf}_{-1}$ & $\begin{array}{c}\text { rate of photoinactivation of } \\
\text { CRY2_BD }\end{array}$ & 3. $55 \mathrm{E}-05$ & $\mathrm{~m} 2 / \mathrm{um}$ & \\
\hline $\operatorname{trf}_{2}$ & $\begin{array}{c}\text { rate of photoactivation of } \\
\text { CIB1_AD }\end{array}$ & 7. $44 \mathrm{E}-04$ & $\mathrm{~m} 2 / \mathrm{um}$ & \\
\hline $\operatorname{trf} f_{-2}$ & $\begin{array}{c}\text { rate of photoinactivation of } \\
\text { CIB1_AD }\end{array}$ & 1. $70 \mathrm{E}-03$ & $\mathrm{~m} 2 / \mathrm{um}$ & \\
\hline $\operatorname{trlc} c_{1}$ & $\begin{array}{c}\text { translocation rate of } \mathrm{Si}_{-} \text {Tag } \\
\text { to membrane }\end{array}$ & 1. $37 \mathrm{E}+01$ & $\mathrm{nM} / \mathrm{h}$ & \multirow[t]{2}{*}{ estimated } \\
\hline $\operatorname{trl} c_{2}$ & $\begin{array}{c}\text { translocation rate of } \operatorname{Mcfp}-3 \\
\text { to extracellular }\end{array}$ & 1. $52 \mathrm{E}+01$ & $\mathrm{nM} / \mathrm{h}$ & \\
\hline $\operatorname{deg}_{1}$ & rate of mCRY2_BD degradation & $3(12 \mathrm{~min})$ & $1 / \mathrm{h}$ & \multirow[t]{4}{*}[1]{$[3]$} \\
\hline $\operatorname{deg}_{2}$ & rate of CRY2_BD degradation & $0.8(48 \mathrm{~min})$ & $1 / \mathrm{h}$ & \\
\hline $\operatorname{deg}_{3}$ & rate of mCIB1_AD degradation & $3(13 \mathrm{~min})$ & $1 / \mathrm{h}$ & \\
\hline $\operatorname{deg}_{4}$ & rate of CIB1_AD degradation & $0.8(48 \mathrm{~min})$ & $1 / \mathrm{h}$ & \\
\hline $\operatorname{deg}_{7}$ & rate of mSi_Tag degradation & $5(13 \mathrm{~min})$ & $1 / \mathrm{h}$ & \multirow[t]{2}{*}{ estimated } \\
\hline $\operatorname{deg}_{8}$ & rate of $\mathrm{Si}_{-}$Tag degradation & $0.3(48 \mathrm{~min})$ & $1 / \mathrm{h}$ & \\
\hline $\operatorname{deg}_{9}$ & rate of $\mathrm{mMcfp}-3$ degradation & $2(12 \mathrm{~min})$ & $1 / \mathrm{h}$ & \multirow[t]{2}{*}[5]{} \\
\hline $\operatorname{deg}_{10}$ & rate of Mcfp-3 degradation & $0.2(48 \mathrm{~min})$ & $1 / \mathrm{h}$ & \\
\hline $\mathrm{k}_{\mathrm{m} 1}$ & $\begin{array}{l}\text { apparent association constant } \\
\text { for CRY2_BD binding with UAS }\end{array}$ & 4. $93 \mathrm{E}+00$ & $\mathrm{nM}$ & [2] \\
\hline $\mathrm{k}_{\mathrm{m} 2}$ & $\begin{array}{l}\text { apparent association constant } \\
\text { for CIB1_AD binding with }\end{array}$ & 1. $04 \mathrm{E}+00$ & $\mathrm{nM}$ & {$[1][3]$} \\
\hline
\end{tabular}




\begin{tabular}{|c|c|c|c|c|}
\hline & CRY2_BD & & & \\
\hline $\mathbf{T}$ & $\begin{array}{c}\text { time for AD activating } \\
\text { promoter pGal1 }\end{array}$ & 11 & Min & estimated \\
\hline
\end{tabular}

[1] Taslimi A, Zoltowski B, Miranda J G, et al. Optimized second-generation CRY2-CIB dimerizers and photoactivatable Cre recombinase[J]. Nature Chemical Biology, 2016.

[2] Sorokina O, Kapus A, Terecskei K, et al. A switchable light-input, light-output system modelled and constructed in yeast[J]. Journal of biological engineering, 2009, 3(1): 1.

[3] Kennedy M J, Hughes R M, Peteya L A, et al. Rapid blue-light-mediated induction of protein interactions in living cells[J]. Nature methods, 2010, 7(12): 973-975.

[4]Taniguchi K, Nomura K, Hata Y, et al. The Si - tag for immobilizing proteins on a silica surface[J].

Biotechnology and bioengineering, 2007, 96(6): 1023-1029.

[5]Platko J D, Deeg M, Thompson V, et al. Heterologous expression of Mytilus californianus foot protein three (Mcfp-3) in Kluyveromyces lactis[J]. Protein expression and purification, 2008, 57(1): 57-62.

\section{Modeling Part one: The Light Control System}

First of all, we intended to simulate the whole pathway to approximate the final expression rate of target proteins as well as the property of the light induction system. To achieve this, we need to translate our biological processes to chemical reactions and finally represent it with mathematical equations. The Light Induction System pathway contains the following reactions:

\section{Formulary}

1. Generation of active CIB1_AD

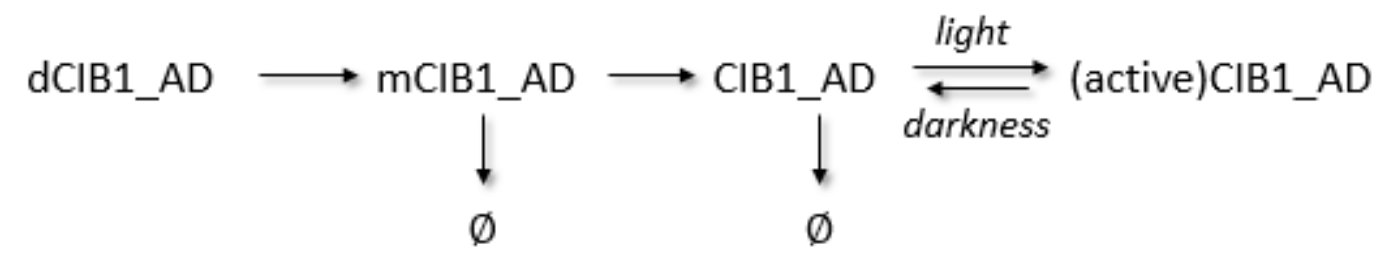

2. Generation of active CRY2_BD

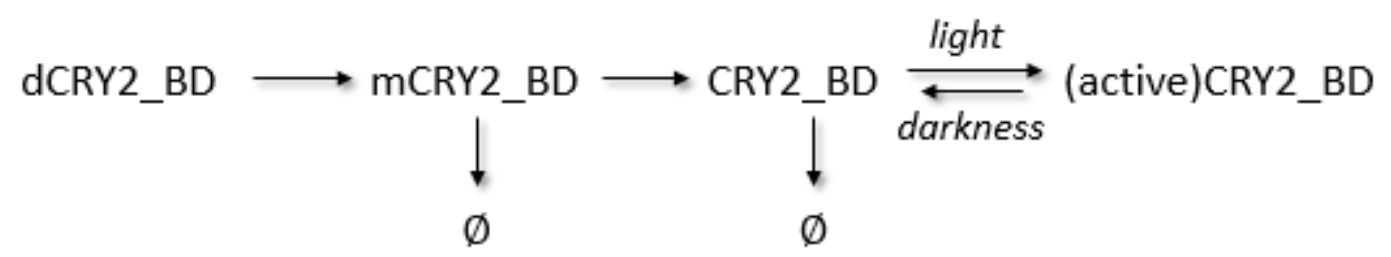

3. Activation of promoter Gal1 
$($ active $)$ CIB1_AD
+
UAS + (active)CYR2_BD $\longrightarrow$ UAS_BD_CRY2 $\longrightarrow$ UAS_BD_CRY2_CIB1_AD

The corresponding DDEs of light induction system are listed below:

1. Generation of CIB1_AD

$$
\begin{aligned}
& \frac{d\left[m C I B 1_{-} A D\right]}{d t}=\operatorname{trc}_{2} * \operatorname{copynum}_{p R M H 120}-\operatorname{deg}_{3} *\left[m C I B 1_{-} A D\right] \\
& \frac{d\left[C I B 1_{-} A D\right]}{d t}=\operatorname{trl} l_{2} *\left[m C I B 1_{-} A D\right]-\operatorname{deg}_{4} *\left[C I B 1_{-} A D\right] \\
& \frac{d\left[(\text { active }) C I B 1_{-} A D\right]}{d t}=\operatorname{trf} 2_{2} *\left[C I B 1_{-} A D\right]-\operatorname{trf} f_{-2} *\left[(\text { active }) C I B 1_{-} A D\right]
\end{aligned}
$$

2. Generation of CRY2_BD

$$
\begin{aligned}
& \frac{d\left[m C R Y 2_{-} B D\right]}{d t}=\operatorname{trc} c_{1} * \text { copynum }_{p R M H 120}-\operatorname{deg}_{1} *\left[m C R Y 2_{-} B D\right] \\
& \frac{d\left[C R Y 2_{-} B D\right]}{d t}=\operatorname{trl} l_{1}^{*}\left[m C R Y 2_{-} B D\right]-d e g_{2} *\left[C R Y 2_{-} B D\right] \\
& \frac{d\left[(\text { active }) C R Y 2_{-} B D\right]}{d t}=\operatorname{trf} f_{1} *\left[C R Y 2_{-} B D\right]-\operatorname{trf} f_{-1} *\left[(\text { active }) C R Y 2_{-} B D\right]
\end{aligned}
$$

3. Activation of promoter Gal1

$$
\left[U A S_{-} B D_{-} C R Y 2\right]=\text { copynum }_{p R M H 120} * \frac{\left[(\text { active }) C R Y 2_{-} B D\right]}{\left[(\text { active }) C R Y 2_{-} B D\right]+k_{m 1}}
$$

If exposed to light

$$
\left[U A S_{-} B D_{-} C R Y 2_{-} C I B 1\right]=\left[U A S_{-} B D_{-} C R Y 2\right]^{*} \frac{\left[(\text { active }) C I B 1_{-} A D\right]}{\left[(\text { active }) C I B 1_{-} A D\right]+k_{m 2}}
$$

Or else if in darkness

$$
\left[U A S_{-} B D_{-} C R Y 2_{-} C I B 1\right]=\left[U A S_{-} B D_{-} C R Y 2\right]^{*} \frac{\left[(\text { active }) C I B 1_{-} A D\left(t-\tau_{1}\right)\right]}{\left[(\text { active }) C I B 1_{-} A D\left(t-\tau_{1}\right)\right]+k_{m 2}}
$$

\section{Results}


Before the circuit was determined, there were two kinds of light induction system for choice: the CRY2-CIB1 system and the PhyA-FHL system. To find out the system that suits our circuit better, we simulated both of them with the DDE model.
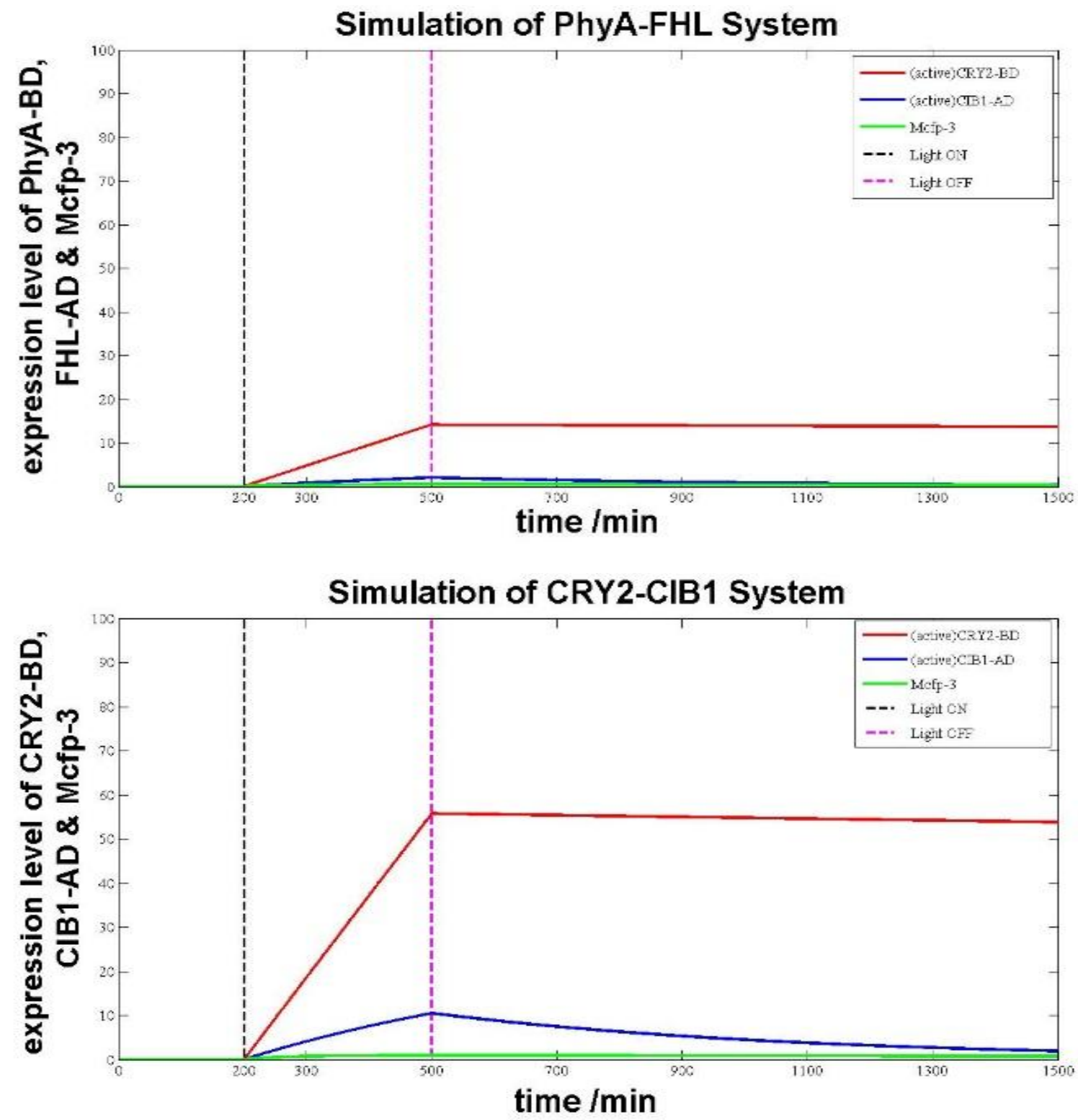

Figure 1.1: Simulation of PhyA-FHL and CRY2-CIB1 system

The following conclusions can be derived from the figure above:

- When exposed to light, concentration of photoactive subjects increases.

- Compared to the PhyA-FHL system, the CRY2-CIB1 system is more sensitive to light exposure because the slope of CRY2-CIB1 system is bigger than the one of PhyA-FHL system.

- Undergoing the same time of light exposure, the accumulated photoactive subjects (active CRY2 \& CIB1) in CRY2-CIB1 system is much more than the ones (active PhyA and FHL) in PhyA-FHL system.

Hence, CRY2-CIB1 system was considered more advantageous and applied it to our project. 


\section{Modeling Part two: The Surface Display System of Si-tag}

With the simulation of Light Control System above, we are able to determine when the Surface Display System of Si-tag would be activated. However, to predict whether Si-tag would be sufficient in the end and to provide essential parameters for the next model, we need to simulate the Surface Display System of Si-tag.

\section{Formulary}

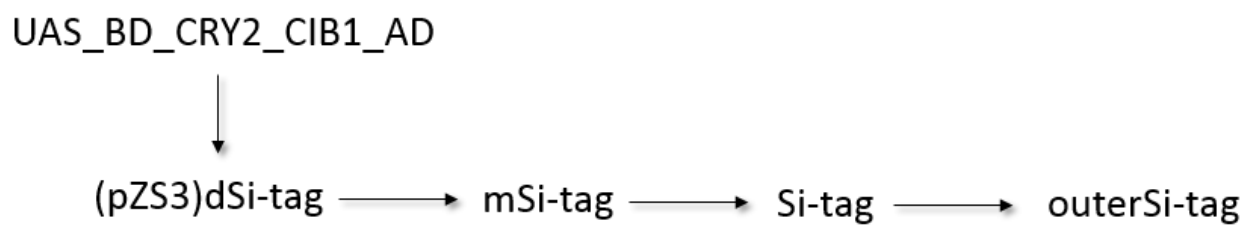

The corresponding DDE of Surface Display System of Si-tag are listed below:

$$
\begin{aligned}
& \frac{d\left[m S i_{-} T a g\right]}{d t}=t r c_{3} *\left[U A S_{-} B D_{-} C R Y 2_{-} C I B 1\right]+ \\
& \operatorname{trc}_{4} *\left(\text { copynum }_{p Z S 3}-\left[U A S_{-} B D_{-} C R Y 2_{-} C I B 1\right]\right)-\operatorname{deg}_{5} *\left[m S i_{-} \text {Tag }\right] \\
& \frac{d\left[S i_{-} T a g\right]}{d t}=\operatorname{trl}_{4} *\left[m S i_{-} T a g\right]-\operatorname{deg}_{6} *\left[S i_{-} \text {Tag }\right]-t r l c_{1} *\left[S i_{-} \text {Tag }\right] \\
& \frac{d[\text { outerSi_Tag }]}{d t}=\operatorname{trlc}_{1} *\left[S i_{-} \text {Tag }\right]
\end{aligned}
$$

\section{Results}

With the DDE model built, we could run the simulation of the expression of Si-tag and determine its amount at any time. To test the function of our darkness induction system, the timeline would be set as darkness-light-darkness. 


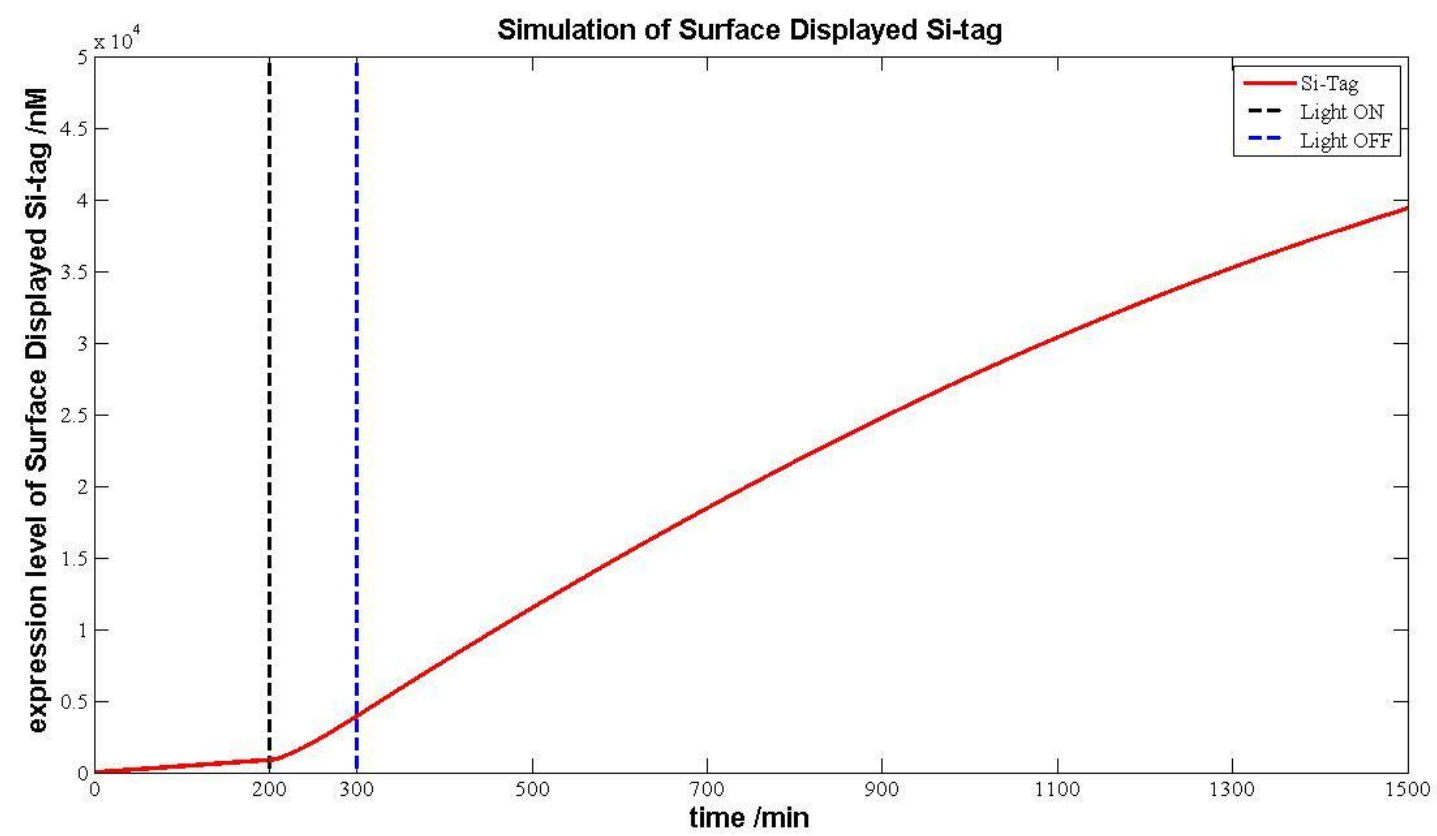

Figure 2: Simulation of Surface Display System of Si-tag

From figure 2, we can see that the Displayed Si-tag accumulates slowly when Euk.Cement is in darkness for the first time (0-200min). However, when exposed to light (200-300min), the rate of Displayed Si-tag accumulation increases greatly. After light exposure (300-1500min), the rate of Displayed Si-tag accumulation gradually recover. Generally speaking, the light induction system is capable of controlling the downstream system and the expression of Si-tag is sufficient.

\section{Modeling Part three: The Expression of Mcfp-3}

Besides Si-tag, MCFP3 is another important product that we must quantify its amount. Its promoter is the same with the promoter of Si-tag. Therefore, we could easily simulate it with the DDE model and MATLAB.

\section{Formulary}

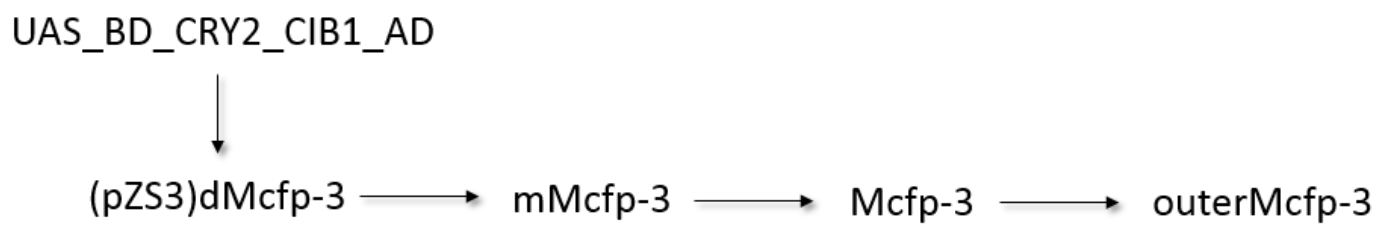

The corresponding DDE of the expression of Mcfp-3 are listed below 


$$
\begin{aligned}
& \frac{d\left[m M c f p_{-} 3\right]}{d t}=\operatorname{trc}_{5} *\left[U A S_{-} B D_{-} C R Y 2_{-} C I B 1\right]+ \\
& \operatorname{trc}_{6} *\left(\text { copynum }_{p Z \mathrm{ZS} 3}-\left[U A S_{-} B D_{-} C R Y 2_{-} C I B 1\right]\right)-d e g_{7} *\left[m M c f p_{-} 3\right] \\
& \frac{d\left[M c f p_{-} 3\right]}{d t}=t r l_{5} *\left[m M c f p_{-} 3\right]-\operatorname{deg}_{8} *\left[M c f p_{-} 3\right]-t r l c_{2} *\left[M c f p_{-} 3\right] \\
& \frac{d[\text { outerMcfp_3 } 3]}{d t}=\operatorname{trlc}_{2} *\left[M_{c} f p_{-} 3\right]
\end{aligned}
$$

\section{Results}

To test the light induction system again as well as to quantify the amount of Mcfp-3, we set the same timeline with that of Si-tag and run the simulation with MATLAB.

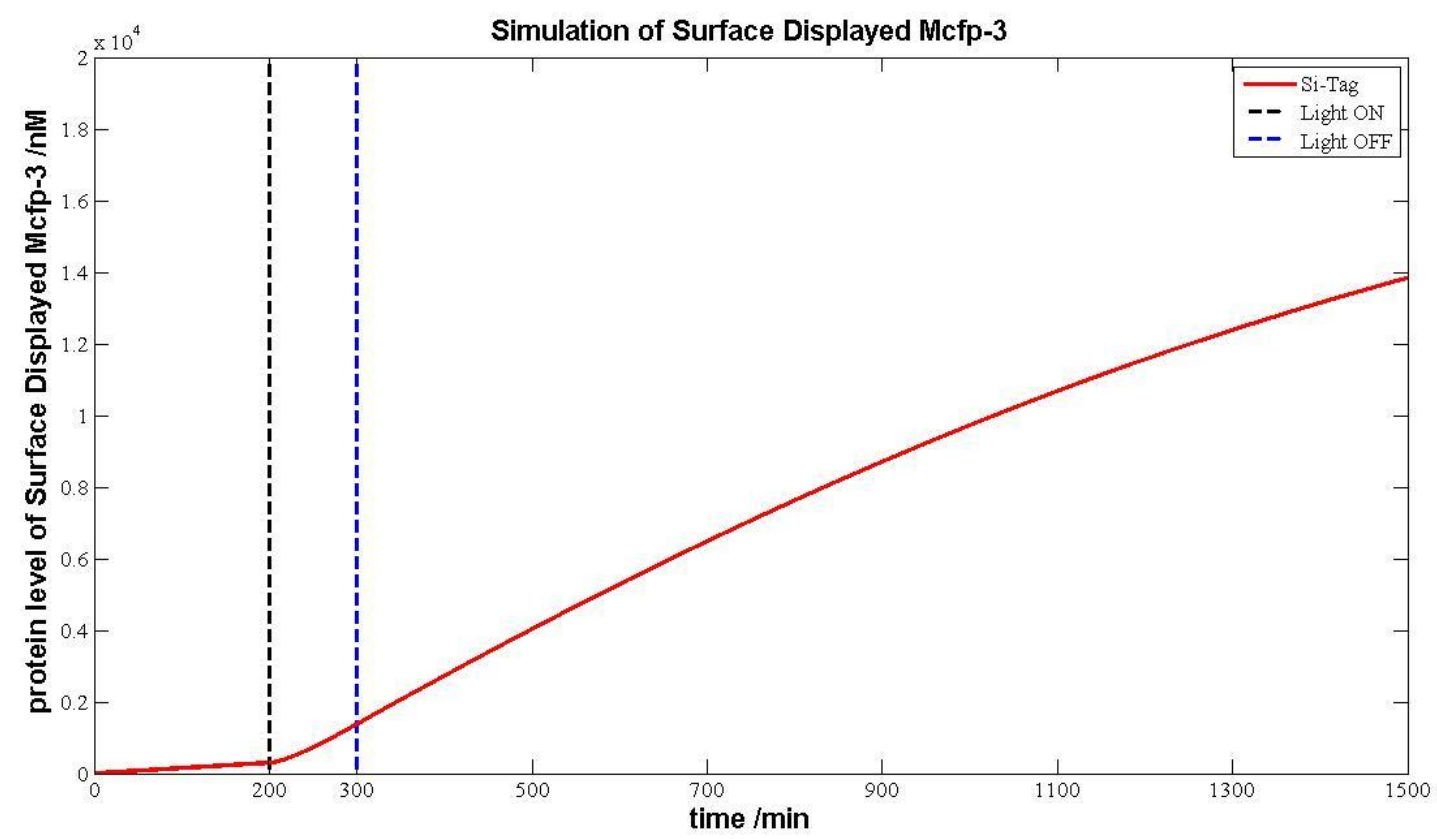

Figure 3: Simulation of the expression of Mcfp-3

As we can see in figure 2 and 3, the amount of Si-tag was approximately twice as Mcfp-3. Besides, the light induction system took effect again. At 200 minutes when the light is turned on, the slope of Si-tag and Mcfp-3 increase significantly. After 300 minutes when the light is turned off, the slope of these two substances gradually decrease again.

\section{Conclusions}

With our DDE model, we can draw some conclusions that:

- Our light induction system could switch efficiently from darkness to light to activate the downstream systems. However, it takes a long time to switch back from light to darkness. 
- Our strains could produce sufficient Si-tag as well as Mcfp-3 to do the job.

- We can quantify the amount of Si-tag and Mcfp-3 at any time so that we can move on to the next model with these data.

\section{Robustness and Parameter Sensitivity Analysis}

Considering there are some parameters whose value is uncertain and may have effect on our model, we used numerical solutions to analyze the robustness and parameter sensitivity of our DDE model.

\section{$\tau$}

Since $\tau$ is a variable that we could not determine their accurate values (0.1-3min), we run the DDE model with different values of $\tau$ to find out what effect they have on our DDE model.
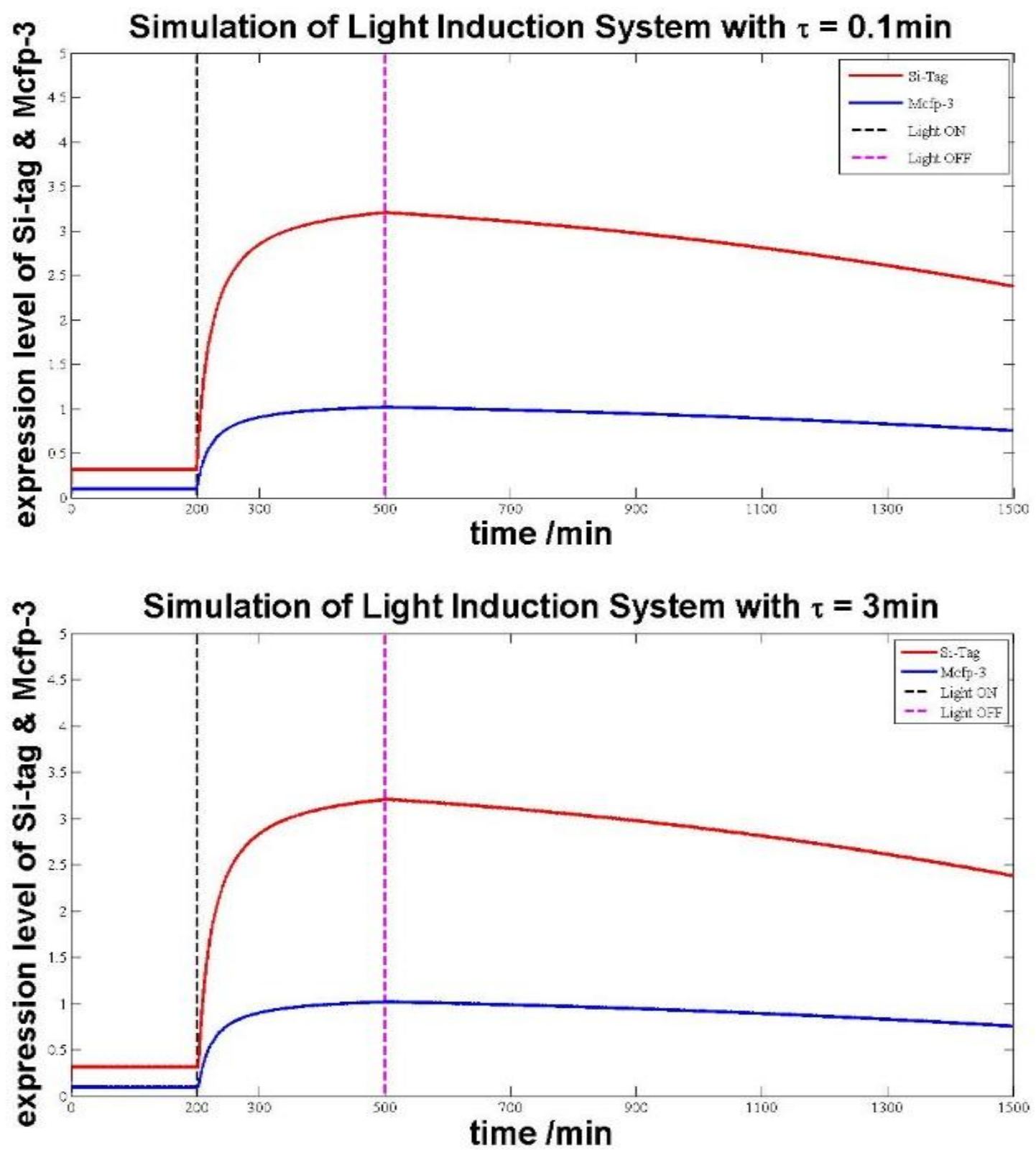

Figure 4: Simulation with different $\tau$ 


\section{Results}

Apparently, even the max value of $\tau$ couldn't produce noticeable time-lag for the whole system, because the time-lag produced by $\tau$ is approximately 5 minutes while the whole simulation process has the timeline of several thousands of minutes. Therefore, we can conclude that our DDE model has the robustness of $\tau$. 